\title{
Influência do Propofol e do Etomidato no Bloqueio Neuromuscular Produzido pelo Rocurônio. Avaliação pela Aceleromiografia *
}

\section{Influence of Propofol and Etomidate on Rocuronium-Induced Neuromuscular Block. Evaluation with Acceleromyography}

\author{
Derli Conceição Munhoz ${ }^{1}$, Angélica de Fátima de Assunção Braga, TSA ${ }^{2}$, Glória Maria Braga Potério, TSA $^{2}$
}

\section{RESUMO}

Munhoz DC, Braga AFA, Potério GMB - Influência do Propofol e do Etomidato no Bloqueio Neuromuscular Produzido pelo Rocurônio. Avaliação pela Aceleromiografia

Justificativa e Objetivos - Alguns hipnóticos podem interagir com os bloqueadores neuromusculares (BNM) potencializando seus efeitos. O objetivo deste estudo foi avaliar a influência do propofol e do etomidato sobre o bloqueio neuromuscular produzido pelo rocurônio.

Método - Foram incluídos no estudo 60 pacientes, estado físico ASA I e II, submetidos a cirurgias eletivas sob anestesia geral, distribuídos aleatoriamente em dois grupos de acordo com o hipnótico empregado: Grupo I (propofol) e Grupo II (etomidato). Todos os pacientes receberam midazolam $\left(0,1 \mathrm{mg}^{\mathrm{kg}} \mathrm{k}^{-1}\right)$ por via muscular como medicação pré-anestésica, 30 minutos antes da cirurgia. A indução anestésica foi obtida com propofol $\left(2,5 \mathrm{mg} \cdot \mathrm{kg}^{-1}\right)$ ou etomidato $\left(0,3 \mathrm{mg} \cdot \mathrm{kg}^{-1}\right)$ precedido de alfentanil $\left(50 \mu \mathrm{g} \cdot \mathrm{kg}^{-1}\right)$ e seguido de rocurônio $\left(0,6 \mathrm{mg} \cdot \mathrm{kg}^{-1}\right)$. Os pacientes foram ventilados sob máscara com oxigênio a $100 \%$ até a obtenção de redução de $75 \%$ ou mais na amplitude da resposta do músculo adutor do polegar, quando foram realizadas as manobras de laringoscopia e intubação traqueal. A função neuromuscular foi monitorizada com aceleromiografia. Foram avaliados: tempo de início de ação do rocurônio ( $\left.T_{1} \leq 25 \%\right)$; tempo para instalação do bloqueio neuromuscular total; grau de bloqueio neuromuscular no momento da intubação traqueal; condições de intubação traqueal e repercussões hemodinâmicas.

Resultados - Os tempos de início de ação e instalação de bloqueio neuromuscular total (segundos) produzido pelo rocurônio foram: Grupo I $(48,20 \pm 10,85$ s e $58,87 \pm 10,73$ s) e Grupo II $(51,20 \pm 13,80$ s e $64,27 \pm 18,55$ s). O grau de bloqueio neuromuscular no momento da intubação traqueal foi: Grupo I $(77,50 \%)$ e Grupo II $(76,96 \%)$. As condições de intubação traqueal foram satisfatórias em $100 \%$ dos pacientes do Grupo I e em $83,33 \%$ no Grupo II. Nos dois grupos, após a injeção do hipnótico, observou-se diminuição significativa da pressão arterial média seguida de elevação.

\footnotetext{
* Recebido do (Received from) Departamento de Anestesiologia da Faculdade de Ciências Médicas (FCM - UNICAMP), Campinas, SP

1. Anestesiologista do Hospital de Clínicas da UNICAMP

2. Profa. Dr ${ }^{a}$. do Departamento de Anestesiologia da FCM - UNICAMP
}

Apresentado (Submitted) em 25 de fevereiro de 2002

Aceito (Accepted) publicação em 30 de abril de 2002

Correspondência para (Mail to):

Dra. Derli da Conceição Munhoz

Rua Votorantim, 51/21 - Vila Nova

13073-090 Campinas, SP

(c) Sociedade Brasileira de Anestesiologia, 2002

Revista Brasileira de Anestesiologia

Vol. 52, № 6, Novembro - Dezembro, 2002
Conclusões - O propofol e o etomidato comportaram-se de maneira semelhante em relação à instalação do bloqueio neuromuscular e às condições de intubação traqueal produzidos pelo rocurônio.

UNITERMOS: BLOQUEADORES NEUROMUSCULARES, Não-Despolarizantes: rocurônio; HIPNÓTICOS: etomidato, propofol; TÉCNICAS DE MEDIÇÃO: aceleromiografia

\section{SUMMARY}

Munhoz DC, Braga AFA, Potério GMB - Influence of Propofol and Etomidate on Rocuronium-Induced Neuromuscular Block. Evaluation with Acceleromyography

Background and Objectives - Some hypnotics may interact with neuromuscular blockers and potentiate their effects. This study aimed at evaluating the influence of propofol and etomidate on rocuronium-induced neuromuscular block.

Methods - Participated in this study 60 patients, physical status ASA I and II, scheduled for elective surgeries under general anesthesia, who were randomly distributed in two groups according to the hypnotic drug: Group I (propofol) and Group II (etomidate). All patients were premedicated with intramuscular midazolam $\left(0.1 \mathrm{mg}^{\mathrm{kg}} \mathrm{g}^{-1}\right), 30$ minutes before surgery. Anesthesia was induced with propofol $\left(2.5 \mathrm{mg} \cdot \mathrm{kg}^{-1}\right)$ or etomidate $(0.3$ $\left.\mathrm{mg} \cdot \mathrm{kg}^{-1}\right)$ preceded by alfentanil $\left(50 \mu \mathrm{g} \cdot \mathrm{kg}^{-1}\right)$ and followed by rocuronium $\left(0.6 \mathrm{mg}^{\mathrm{kg}} \mathrm{k}^{-1}\right)$. Patients were ventilated under mask with $100 \%$ oxygen until achieving a decrease of $75 \%$ or more in the adductor pollicis muscle response amplitude. Neuromuscular function was monitored by accelerometry. The following parameters were evaluated: rocuronium onset $\left(T_{1} \leq\right.$ 25\%); time for complete neuromuscular block; neuromuscular block degree at tracheal intubation; tracheal intubation conditions and hemodynamic effects.

Results - Complete rocuronium-induced neuromuscular block onset times (in seconds) were: Group I (48.20 $\pm 10.85 \mathrm{~s}$ and $58.87 \pm 10.73 \mathrm{~s}$ ) and Group II (51.20 $\pm 13.80 \mathrm{~s}$ and $64.27 \pm 18.55$ s). Neuromuscular block degree at tracheal intubation was: Group I (77.50\%) and Group II (76.96\%). Tracheal intubation conditions were satisfactory in $100 \%$ of Group I patients and in 83.33\% of Group II patients. There has been a significant decrease in mean blood pressure, followed by an increase after hypnotic injection, in both groups.

Conclusions - Propofol and etomidate had a similar behavior regarding time for rocuronium-induced neuromuscular block and tracheal intubation conditions.

KEY WORDS: HYPNOTICS: propofol, etomidate; MEASUREMENT TECHNIQUES: acceleromyography; NEUROMUSCULAR BLOCKERS, Nondepolarizing: rocuronium 


\section{INTRODUÇÃO}

$\mathrm{O}$ grande número de bloqueadores neuromusculares (BNM) não despolarizantes disponíveis na clínica tornou o seu uso cada vez mais freqüente em substituição a succinilcolina, na obtenção de adequado relaxamento muscular para as manobras de laringoscopia e intubação traqueal. Pesquisas foram direcionadas para obtenção de BNM adespolarizante, cuja latência fosse semelhante à da succinilcolina, porém sem os efeitos colaterais indesejáveis desta. O rocurônio é um BNM não despolarizante que apresenta rápido início de ação ${ }^{1}$. Técnicas alternativas também foram avaliadas para encurtar o tempo de latência destes agentes; entre elas, o uso de altas doses de um único bloqueador, a combinação de diferentes bloqueadores ou o emprego de doses fracionadas destes agentes ${ }^{2-4}$. Entre os fármacos utilizados na indução anestésica, os hipnóticos podem interferir no relaxamento muscular induzido pelos BNM não despolarizantes ${ }^{5}$. Alguns destes agentes hipnóticos apresentam certas peculiaridades, como o etomidato, que por sua estabilidade hemodinâmica pode contribuir para o menor tempo de latência dos BNM não despolarizantes ${ }^{6}$. O propofol, além de diminuir a reatividade do tônus muscular da laringe e da faringe, possibilitando a intubação traqueal sem o uso de bloqueadores neuromusculares, quando empregado em doses semelhantes às utilizadas na clínica, tem in vitro ação sobre a junção neuromuscular, reduzindo o tempo de abertura do canal do receptor nicotínico muscular ${ }^{7-10}$.

O objetivo deste estudo foi avaliar comparativamente a influência do propofol e do etomidato na instalação do bloqueio neuromuscular, produzido pelo rocurônio.

\section{MÉTODO}

Após aprovação pelo Comitê de Ética do Hospital e consentimento livre e esclarecido dos pacientes, foram incluídos no estudo 60 pacientes, de ambos os sexos, estado físico ASA I ell, com idades entre 17 e 58 anos e peso entre 42 e 88 quilos, selecionados para cirurgias eletivas sob anestesia geral com indicação de intubação traqueal e ventilação controlada mecânica, distribuídos aleatoriamente em dois grupos, de acordo com o hipnótico empregado, para a indução da anestesia: Grupo I - propofol $(n=30)$ e Grupo II - etomidato $(n=$ 30 ). Nos dois grupos o rocurônio foi administrado na dose de $0,6 \mathrm{mg} \cdot \mathrm{kg}^{-1}$, correspondendo a duas vezes a DE ${ }_{95}$. Constituíram critérios de exclusão pacientes portadores de doenças neuromusculares, renais ou hepáticas, alterações hidroeletrolíticas e ácido-base, história de refluxo gastroesofágico, em uso de drogas que interagem com os bloqueadores neuromusculares, e com sinais indicativos de dificuldades para a realização das manobras de laringoscopia e intubação traqueal (Mallampati III e IV ${ }^{11}$ ). A medicação pré-anestésica consistiu de midazolam $\left(0,1 \mathrm{mg} \cdot \mathrm{kg}^{-1}\right)$, por via muscular, 30 minutos antes da indução anestésica. Na sala cirúrgica, uma veia periférica foi canulizada para hidratação e administração de drogas. Aindução da anestesia foi obtida com alfenta- nil $\left(50 \mu \mathrm{g} \cdot \mathrm{kg}^{-1}\right)$ seguido de propofol $\left(2,5 \mathrm{mg} \cdot \mathrm{kg}^{-1}\right)$ ou etomidato $\left(0,3 \mathrm{mg} \cdot \mathrm{kg}^{-1}\right)$ e rocurônio $\left(0,6 \mathrm{mg} \cdot \mathrm{kg}^{-1}\right)$. Os pacientes foram ventilados sob máscara com oxigênio a $100 \%$, realizando-se as manobras de laringoscopia e intubação traqueal após obtenção da redução de $75 \%$ ou mais na amplitude da resposta controle no músculo adutor do polegar.

Utilizou-se como monitorização contínua o cardioscópio na derivação $D_{\text {Il }}$, oxímetro de pulso e monitor não invasivo de pressão arterial. Para a avaliação do bloqueio neuromuscular foi empregado monitor de transmissão neuromuscular (Aceleromiógrafo - TOF-GUARD). Antes da indução anestésica foram aplicados estímulos supramaximais $(1 \mathrm{~Hz})$, durante dez minutos, para a estabilização da resposta controle, empregando-se eletrodos de superfície no trajeto do nervo ulnar, no punho. Um transdutor de aceleração (piezoelétrico) foi fixado na falange distal do polegar do membro monitorizado e um sensor de temperatura sobre a pele na região tenar. Durante e após a indução anestésica a função neuromuscular foi monitorizada continuamente com estímulos isolados até a obtenção de bloqueio neuromuscular total. As respostas do adutor do polegar mostradas em gráficos de barras e em números digitais foram armazenadas em um cartão memória e posteriormente reproduzidas em computador compatível, previamente programado. Nos registros dos traçados das respostas musculares (Figura 1) observam-se: 1) injeção do hipnótico; 2) injeção do rocurônio; 3) início de ação do rocurônio (momento da intubação traqueal); 4) bloqueio neuromuscular total.

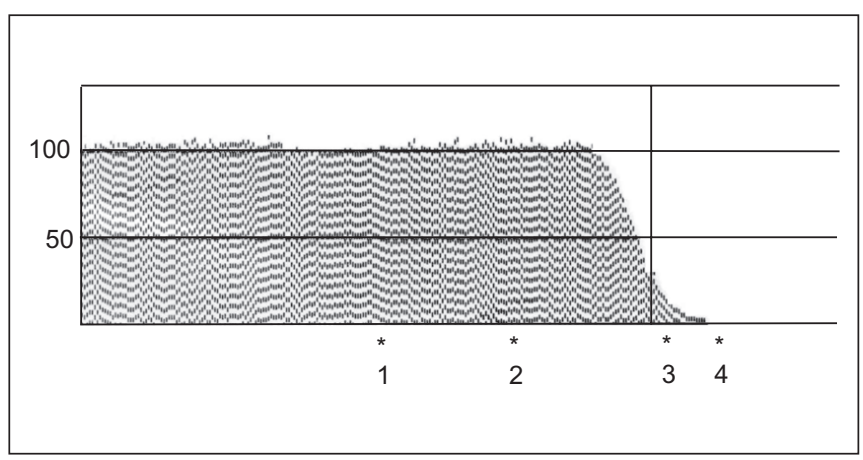

Figura 1 - Respostas do músculo adutor do polegar, 1- injeção do hipnótico; 2- injeção do rocurônio; 3- inicio de ação do rocurônio (momento da intubação traqueal); 4- bloqueio neuromuscular total

Foram avaliados: 1) parâmetros neuromusculares - início de ação do rocurônio: intervalo de tempo (segundos) decorrido entre o início da injeção do rocurônio e a obtenção da redução de $75 \%$ ou mais na amplitude das respostas do músculo adutor do polegar; tempo para bloqueio neuromuscular total: intervalo de tempo (segundos) decorrido entre o início da injeção do rocurônio e a obtenção de $100 \%$ de bloqueio no músculo adutor do polegar; grau de bloqueio neuromuscular no momento da intubação traqueal: redução percentual na amplitude da resposta do músculo adutor do polegar em relação ao momento controle; 2) condições clínicas de intuba-

Revista Brasileira de Anestesiologia Vol. 52, N 6, Novembro - Dezembro, 2002 
ção traqueal, empregando-se o método proposto por Helbon-Hansen e col. (1988) ${ }^{12}$, considerando o grau de dificuldade à laringoscopia, a presença e intensidade de tosse e a posição e movimento das cordas vocais, atribuindo-se valores de um a quatro a cada um destes parâmetros (Tabela I). Pontuação menor ou igual a dois aos três parâmetros correspondeu a condições satisfatórias de intubação traqueal, e pontuações acima de dois a um dos três parâmetros foram consideradas insatisfatórias; 3) parâmetros hemodinâmicos: pressão arterial média (PAM) e freqüência cardíaca (FC), avaliados nos seguintes momentos: imediatamente antes da indução anestésica (M0), após a indução anestésica e antes das manobras de laringoscopia e intubação traqueal (M1) e um minuto após a intubação traqueal (M2).

Tabela I - Parâmetros para Avaliação das Condições de Intubação Traqueal (Helbon-Hansen e col.) ${ }^{12}$

\begin{tabular}{lllll}
\hline & \multicolumn{1}{c}{1} & \multicolumn{2}{c}{2} & \multicolumn{1}{c}{4} \\
\hline Laringoscopia & Fácil & Razoável & Difícil & Impossível \\
Cordas Vocais & Abertas & Com movimento & Fechando * & Fechadas \\
Tosse & Ausente & Leve & Moderada & Intensa \\
\hline
\end{tabular}

* Pregas vocais se fecham ao contato com o tubo traqueal

Para análise estatística empregaram-se ANOVA, teste $t$ de Student, teste Exato de Fisher e teste de Mann-Whitney, adotando-se o nível de significância de $5 \%(p<0,05)$.

\section{RESULTADOS}

Com relação aos dados demográficos dos pacientes, não houve diferença significativa entre os grupos, sendo considerados homogêneos (Tabela II).

Tabela II - Características Demográficas dos Pacientes

\begin{tabular}{lcc}
\hline & $\begin{array}{c}\text { Grupo I } \\
\text { (propofol) }\end{array}$ & $\begin{array}{c}\text { Grupo II } \\
\text { (etomidato) }\end{array}$ \\
\hline Idade (anos) & $39,43 \pm 10,20$ & $38,83 \pm 9,91$ \\
Peso $(\mathrm{kg})^{*}$ & $64,03 \pm 11,42$ & $60,43 \pm 10,73$ \\
Estado Físico (ASA I:II)** & $18: 12$ & $16: 14$ \\
Sexo $(\mathrm{M}: \mathrm{F})^{* *}$ & $16: 14$ & $15: 15$ \\
\hline
\end{tabular}

* Dados expressos pela Média \pm DP

** número de pacientes

Tabela IV - Parâmetros Hemodinâmicos (Média — DP)

\begin{tabular}{|c|c|c|c|c|c|c|}
\hline & \multicolumn{3}{|c|}{ Grupo I (propofol) } & \multicolumn{3}{|c|}{ Grupo II (etomidato) } \\
\hline & Mo & M1 & M2 & MO & M1 & M2 \\
\hline PAM (mmHg) & $93,4 \pm 15,3$ & $78,4 \pm 12,0$ & $83,2 \pm 17,5$ & $97,4 \pm 12,5$ & $81,8 \pm 12,1$ & $94,6 \pm 16,9$ \\
\hline $\mathrm{FC}(\mathrm{bpm})$ & $78,2 \pm 15,0$ & $72,2 \pm 12,1$ & $79,0 \pm 15,3$ & $79,0 \pm 12,5$ & $69,2 \pm 14,4$ & $79,3 \pm 21,3$ \\
\hline
\end{tabular}

Quanto ao tempos de início de ação e para bloqueio neuromuscular total, grau de bloqueio neuromuscular no momento da intubação traqueal, não houve diferença significativa entre os grupos. Os valores médios e desvios padrão destas variáveis para os dois grupos encontram-se na tabela III.

Tabela III - Parâmetros Neuromusculares (Média \pm DP)

\begin{tabular}{lcc}
\hline & $\begin{array}{c}\text { Grupo I } \\
\text { (propofol) }\end{array}$ & $\begin{array}{c}\text { Grupo II } \\
\text { (etomidato) }\end{array}$ \\
\hline Início de ação (seg) & $48,20 \pm 10,85$ & $51,20 \pm 13,80$ \\
$\begin{array}{l}\text { Tempo para bloqueio } \\
\text { neuromuscular total (seg) }\end{array}$ & $58,87 \pm 10,73$ & $64,27 \pm 18,55$ \\
$\begin{array}{l}\text { Grau de bloqueio no momento } \\
\text { da intubação traqueal (\%) }\end{array}$ & $77,5 \pm 2,87$ & $77,0 \pm 1,49$ \\
\hline
\end{tabular}

As condições clínicas de intubação traqueal foram consideradas satisfatórias em todos os pacientes do Grupo I. No Grupo II foram satisfatórias em 25 pacientes $(83,33 \%)$ e insatisfatórias em 5 pacientes $(16,66 \%)$. Nos dois grupos, em relação à laringoscopia, posição e movimento das cordas vocais atribuiu-se pontuação 1 a todos os pacientes. Quanto ao parâmetro presença e intensidade de tosse, no Grupo I, 28 pacientes $(93,33 \%)$ apresentaram pontuação 1 e dois pacientes $(6,66 \%)$ receberam pontuação 2 , enquanto no Grupo II, $21(70 \%), 4(13,33 \%)$ e $5(16,66 \%)$ pacientes receberam pontuações 1,2 e 3, respectivamente.

Os valores médios e desvios padrão dos parâmetros cardiocirculatórios nos grupos I e II encontram-se na tabela IV. Nos dois grupos, houve diminuição significante da PAM, quando foram comparados os valores médios entre os temsignificante da PAM entre M1 e M2 $(p<0,05)$. Quanto à freqüência cardíaca, não foram observadas alterações significantes nos diferentes tempos estudados, nos dois grupos.

\section{DISCUSSÃO}

O início de ação de um bloqueador neuromuscular pode ser definido como o intervalo entre o final da injeção do BNM e a depressão máxima da resposta de um músculo periférico, sendo o adutor do polegar o mais freqüentemente empregado na prática clínica. Este tempo pode ser influenciado por alguns fatores, tais como, agentes hipnóticos, tempo para estabilização da resposta muscular controle e freqüência de estímulo utilizada ${ }^{6,13,14}$. Autores observaram experimentalmente que o início de ação de um BNM não despolarizante é inversamente proporcional ao tempo para estabilização da pos M0 e M1 ( $p<0,05)$. No Grupo II, observou-se elevação 
resposta muscular controle e a freqüência do estímulo, sendo portanto mais curto quanto maior o período de estabilização e a freqüência de estímulo aplicada na obtenção desta resposta, antes da administração do $\mathrm{BNM}^{13,14}$. A explicação mais provável é que a sucessão de contrações provocadas pela estimulação do nervo, mantida por um tempo mais prolongado, aumenta o fluxo sangüíneo muscular resultando em maior aporte de droga para o músculo estimulado ${ }^{15,16}$. Uma outra explicação é que altas freqüências de estímulos podem levar a depleção do neurotransmissor no local da estimulação, com conseqüente encurtamento da latência e aumento do grau de bloqueio neuromuscular. Adessensibilização de receptores provocada pela estimulação prévia à administração dos BNM não despolarizantes também pode ser considerada como responsável pela menor latência ${ }^{14}$.

Fatores relacionados à monitorização de bloqueio neuromuscular empregada também interferem no tempo de instalação do bloqueio. O emprego da pré-carga no adutor do polegar, indispensável na mecanomiografia, aumenta o metabolismo regional, levando maior aporte sangüíneo ao músculo monitorizado e, consequentemente, um maior número de moléculas do BNM pode alcançar a junção neuromuscular em menor intervalo de tempo ${ }^{13,14,16}$

Neste estudo utilizou-se o método aceleromiográfico, que prescinde da pré-carga, buscando atenuar a influência da monitorização sobre a instalação do bloqueio. Optou-se pela estimulação do nervo ulnar e avaliação das respostas do músculo adutor do polegar, tendo em vista a sua inervação única e a semelhança do conjunto nervo ulnar-músculo adutor do polegar, com as preparações isoladas nervo-músculo, usadas com muita freqüência nos estudos experimentais ${ }^{17}$. No entanto, é importante ressaltar que, quando se avalia condições de intubação traqueal, a monitorização do adutor do polegar é questionada, visto que a sensibilidade deste músculo aos BNM não despolarizantes não apresenta paralelismo com a dos músculos envolvidos nas manobras de laringoscopia e intubação traqueal ${ }^{18}$.

A maioria dos autores correlaciona a influência dos agentes hipnóticos na latência do bloqueio neuromuscular com a estabilidade hemodinâmica e, indiretamente, com o grau de perfusão muscular. $\mathrm{O}$ agente hipnótico escolhido para a indução anestésica influencia nas condições de intubação traqueal de várias maneiras: por depressão de reflexos laríngeos e faríngeos aos estímulos da intubação traqueal e também por potencializarem os efeitos de alguns bloqueadores neuromusculares ${ }^{19-21}$

Dentre os agentes hipnóticos o propofol é amplamente empregado para a indução de anestesias, por deprimir de maneira significativa os reflexos laríngeos e faríngeos, podendo até mesmo prescindir da ação complementar dos BNM não despolarizantes para a intubação traqueal. Esse efeito evidencia a superioridade do propofol em relação a outros hipnóticos, exteriorizada pela maior incidência de imobilidade das cordas vocais, vista até mesmo nos ensaios nos quais os BNM não despolarizantes não foram utilizados ${ }^{9,10,22-25}$. Sparr e col. ${ }^{26}$ relataram condições aceitáveis de intubação traqueal em $98 \%$ dos pacientes após o emprego de propofol e rocurônio, enquanto que no grupo do tiopental estas foram observadas em $80 \%$ dos casos. Resultados contrários foram descritos por outros autores ${ }^{27}$, que encontraram resultados semelhantes para os dois agentes, em relação as condições de intubação e a instalação do bloqueio produzido pelo rocurônio.

Neste estudo, as condições de intubação traqueal foram satisfatórias em todos os pacientes do grupo do propofol, obtidas num tempo médio de 48,20 segundos, resultados semeIhantes aos de outros autores ${ }^{28}$.

Estudos sobre a influência dos hipnóticos na latência dos bloqueadores neuromusculares realizados com o vecurônio resultaram em latência menor com o etomidato em relação ao tiopental e ao propofol, o que pode ser atribuído ao melhor perfil hemodinâmico do etomidato ${ }^{6}$. Em relação ao rocurônio, resultados semelhantes foram observados com o etomidato e tiopental; no entanto, houve menor incidência de reação diafragmática nos pacientes do grupo do etomidato ${ }^{20}$. No presente trabalho, observaram-se tosse e movimentação diafragmática após a intubação traqueal, com os dois hipnóticos. Esta pode ser explicada pela maior latência do rocurônio no diafragma, em relação à observada nos músculos periféricos ${ }^{29}$. Todos os pacientes foram intubados em um tempo menor que 60 segundos $(48,20$ versus 51,20 segundos), momento em que foi obtido bloqueio neuromuscular maior ou igual a $75 \%$ no músculo adutor do polegar, estipulado previamente como tempo de latência. Amenor incidência de tosse no grupo do propofol (dois pacientes) em relação ao grupo do etomidato, em que quatro pacientes apresentaram tosse leve e cinco, tosse moderada, pode ser devida a maior ação depressora sobre os reflexos laríngeos e faríngeos apresentada pelo propofol ${ }^{30}$.

Em relação aos parâmetros hemodinâmicos, apesar da diminuição significante da PAM, observada após a administração dos agentes hipnóticos, vale salientar que esta não repercutiu sobre a latência do rocurônio e o grau de bloqueio neuromuscular, uma vez que os tempos para obtenção de bloqueio adequado para intubação traqueal e para o bloqueio neuromuscular total foram equivalentes com os dois hipnóticos.

Concluímos que o propofol e o etomidato comportaram-se de maneira semelhante em relação à instalação do bloqueio neuromuscular e às condições de intubação traqueal produzidas pelo rocurônio. 


\section{Influence of Propofol and Etomidate on Rocuronium-Induced Neuromuscular Block. Evaluation with Acceleromyography}

Derli Conceição Munhoz, M.D., Angélica de Fátima de Assunção Braga, TSA, M.D., Glória Maria Braga Potério, TSA, M.D.

\section{INTRODUCTION}

The large number of available nondepolarizing neuromuscular blockers (NMB) has made them increasingly popular in replacing succinylcholine for adequate muscle relaxation during laryngoscopy and tracheal intubation. Research has been targeted to nondepolarizing NMBs with similar onset to succinylcholine but without its side-effects. Rocuronium is a fast onset nondepolarizing NMB ${ }^{1}$. Alternative techniques have also been evaluated to shorten such agents' onset, among them high doses of a single blocker, combination of different blockers or fractionated doses of such agents ${ }^{2-4}$. Hypnotics used in anesthetic induction may interfere with non polarizing NMB-induced neuromuscular relaxation ${ }^{5}$. Some hypnotics, such as etomidate, have certain peculiarities which, due to their hemodynamic stability, may contribute for a shorter non-depolarizing NMB onset ${ }^{6}$. Propofol, in addition to decreasing laryngeal and pharyngeal muscle tone reactivity allowing tracheal intubation without neuromuscular blockers when used in doses similar to those used in the clinic, has an in vitro action on the neuromuscular junction and decreases muscle nicotinic receptor channel opening ${ }^{7-10}$. This study aimed at comparing the influence of propofol and etomidate in rocuronium-induced neuromuscular block.

\section{METHODS}

After the Hospital's Ethical Committee approval and their informed consent, participated in this study 60 patients of both genders, physical status ASA I and II, aged 17 to 58 years, weighing 42 to $88 \mathrm{~kg}$, scheduled for elective surgeries under general anesthesia with indication for tracheal intubation and mechanically controlled ventilation. Patients were divided in two groups, according to the hypnotic agent used for anesthetic induction: Group I - propofol $(n=30)$ and Group II etomidate $(n=30)$. Both groups received $0.6 \mathrm{mg} \cdot \mathrm{kg}^{-1}$ rocuronium or twice $\mathrm{DE}_{95}$. Exclusion criteria were patients with neuromuscular, renal or liver diseases, hydroelectric and acid-base changes, history of gastroesophageal reflux, use of drugs interacting with neuromuscular blockers, and evidences of difficult laryngoscopy and tracheal intubation (Mallampati III and IV ${ }^{11}$ ). Patients were premedicated with 0.1 $\mathrm{mg} . \mathrm{kg}^{-1}$ muscular midazolam 30 minutes before anesthetic induction. In the operating room, a catheter was inserted in a peripheral vein for hydration and drug administration. Anesthesia was induced with alfentanil $\left(50 \mu \mathrm{g} . \mathrm{kg}^{-1}\right)$, followed by propofol $\left(2.5 \mathrm{mg} . \mathrm{kg}^{-1}\right)$ or etomidate $\left(0.3 \mathrm{mg} . \mathrm{kg}^{-1}\right)$ and rocuronium $\left(0.6 \mathrm{mg} \mathrm{kg}^{-1}\right)$. Patients were ventilated under mask with
$100 \%$ oxygen and laryngoscopy and tracheal intubation were performed after $75 \%$ or more decrease in the adductor pollicis muscle response amplitude.

Monitoring consisted of cardioscope at $D_{\|}$, pulse oximeter and non-invasive blood pressure. A neuromuscular transmission monitor was used to evaluate neuromuscular block (Acceleromyograph - TOF-GUARD). Supramaximal stimulations $(1 \mathrm{~Hz})$ were applied before anesthetic induction for 10 minutes to stabilize control-response using surface leads on the ulnar nerve pathway on the wrist. An acceleration transducer (piezoelectric) was placed on the distal phalange of the monitored limb thumb and a temperature sensor was placed on the cutaneous tenar region. Neuromuscular function was continuously monitored during and after anesthetic induction with isolated stimulations until complete neuromuscular block. Adductor pollicis response values in bar charts and digital figures were stored in a memory card and then reproduced in a previously programmed compatible computer. Muscle response plots (Figure 1) show: 1) hypnotic injection; 2) rocuronium injection; 3 ) rocuronium onset (tracheal intubation moment); 4) complete neuromuscular block.

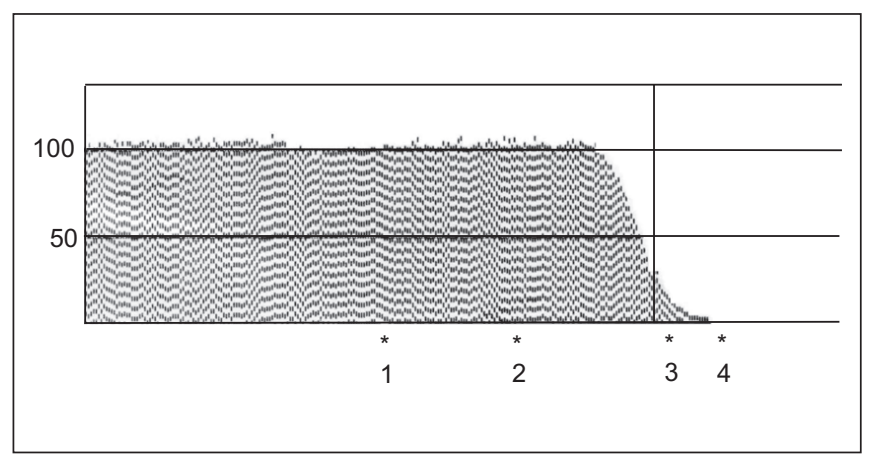

Figure 1 - Adductor pollicis muscle responses, 1 - hypnotic injection; 2 - rocuronium injection; 3 - rocuronium onset (tracheal intubation moment); 4 - complete neuromuscular blockade

The following were evaluated: 1) neuromuscular parameters - rocuronium onset: time (seconds) between beginning of rocuronium injection and $75 \%$ or more decrease in adductor pollicis muscle response amplitude; time for complete neuromuscular block: interval (seconds) between beginning of rocuronium injection and $100 \%$ adductor pollicis muscle blockade; neuromuscular blockade degree at tracheal intubation: percent decrease in adductor pollicis muscle response amplitude as compared to control moment; 2) tracheal intubation clinical conditions using the method proposed by Helbon-Hansen et al. (1988) ${ }^{12}$, considering level of laryngoscopy difficulty, presence and intensity of cough and vocal cords positioning and movement, using a scale from 1 to 4 (Table I). When the three parameters were grade 2 or less, this corresponded to optimal tracheal intubation conditions, and grades above two for one parameter would be considered unsatisfactory; 3 ) hemodynamic parameters: mean blood pressure (MBP) and heart rate (HR), evaluated in the following moments: immediately before anesthetic induction 
(M0), after anesthetic induction and before laryngoscopy and tracheal intubation (M1) and one minute after tracheal intubation (M2).

Table I - Parameters for Tracheal Intubation Conditions Evaluation (Helbon-Hansen et al.) ${ }^{12}$

\begin{tabular}{lllll}
\hline & 1 & \multicolumn{1}{c}{2} & \multicolumn{1}{c}{3} \\
\hline Laryngoscopy & Easy & Reasonable & Difficult & Impossible \\
Vocal cords & Open & With movement & Closing * & Closed \\
Cough & Absent & Mild & Moderate & Severe \\
\hline
\end{tabular}

* Vocal folds close in contact with tracheal tube

ANOVA, Student's $t$, Fisher's Exact and Mann-Whitney tests were used for statistical analysis considering significant $p<$ $0.05(5 \%)$.

\section{RESULTS}

There were no significant differences between groups in demographics data (Table II).

Table II - Demographics Data

\begin{tabular}{|c|c|c|}
\hline & $\begin{array}{c}\text { Group I } \\
\text { (propofol) }\end{array}$ & $\begin{array}{c}\text { Group II } \\
\text { (etomidate) }\end{array}$ \\
\hline Age (years) ${ }^{*}$ & $39.43 \pm 10.20$ & $38.83 \pm 9.91$ \\
\hline Weight $(\mathrm{kg})^{*}$ & $64.03 \pm 11.42$ & $60.43 \pm 10.73$ \\
\hline Physical status (ASA I:II)** & $18: 12$ & $16: 14$ \\
\hline Gender $(\mathrm{M}: \mathrm{F})^{\star *}$ & $16: 14$ & $15: 15$ \\
\hline
\end{tabular}

* Data expressed in Mean \pm SD

** number of patients

There were also no significant differences between groups in onset and complete neuromuscular block time, and neuromuscular block degree at tracheal intubation. Mean and standard deviation of such variables for both groups are shown in table III.

Table III - Neuromuscular Parameters (Mean \pm SD)

\begin{tabular}{lcc}
\hline & $\begin{array}{c}\text { Group I } \\
\text { (propofol) }\end{array}$ & $\begin{array}{c}\text { Group II } \\
\text { (etomidate) }\end{array}$ \\
\hline Onset time (sec) & $48.20 \pm 10.85$ & $51.20 \pm 13.80$ \\
$\begin{array}{l}\text { Time for complete } \\
\text { neuromuscular block (sec) }\end{array}$ & $58.87 \pm 10.73$ & $64.27 \pm 18.55$ \\
$\begin{array}{l}\text { Blockade degree at } \\
\text { tracheal intubation }(\%)\end{array}$ & $77.5 \pm 2.87$ & $77.0 \pm 1.49$ \\
\hline
\end{tabular}

Tracheal intubation clinical conditions were considered satisfactory for all Group I patients. in Group II, they were considered satisfactory in 25 patients (83.33\%) and unsatisfactory in 5 patients $(16.66 \%)$. All patients of both groups had grade 1 for laryngoscopy, vocal cords position and movement. As to the presence and intensity of cough, 28 Group I patients $(93.33 \%)$ received grade 1 and two patients $(6.66 \%)$ grade 2 , while $21(70 \%), 4(13.33 \%)$ and $5(16.66 \%)$ Group II patients received grades 1,2 and 3 , respectively.

Cardiocirculatory parameters mean and standard deviation for both groups are shown in table IV.

There has been a significant MBP decrease in both groups when comparing mean $M 0$ and $M 1$ values $(p<0.05)$. Group II has shown a significant MBP increase between $M 1$ and $M 2$ ( $p$ $<0.05$ ). There were no significant changes in heart rate for both groups in all studied moments.

\section{DISCUSSION}

Neuromuscular blockers onset may be defined as the time elapsed between the end of NMB injection and maximum depression of a peripheral nerve, being the adductor pollicis the most widely used in the clinical practice. This time may be influenced by some factors, such as hypnotic agents, time for muscle control response stabilization and stimulation frequency ${ }^{6,13,14}$. Some authors have experimentally observed that non depolarizing NMBs onset is inversely proportional to the time for muscle control response stabilization and stimulation frequency, thus being faster with longer stabilization and frequency of stimulation applied to obtain such response, before NMB administration ${ }^{13,14}$. The most likely explanation is that the succession of contractions caused by nerve stimulation and kept for a longer time would increase muscle blood blow resulting in more drug transported to the stimulated nerve ${ }^{15,16}$. A different explanation is that high stimulation frequencies may lead to neurotransmitter depletion at the stimulation site, with a consequent faster onset and neuromuscular block degree increase. The de-sensitization of receptors caused by stimulation before non depolarizing NMB administration may also be considered responsible for this faster onset ${ }^{14}$

Monitoring-related factors may also interfere with blockade onset. Adductor pollicis preload, mandatory for mechanomyography, increases regional metabolism sending more blood to the monitored muscle and, as a consequence, more NMB molecules may reach the neuromuscular junction in a shorter period of time ${ }^{13,14,16}$.

Our study has used acceleromyography, which does not need preload, in an attempt to attenuate the influence of mo-

Table IV - Hemodynamic Parameters (Mean \pm SD)

\begin{tabular}{|c|c|c|c|c|c|c|}
\hline & \multicolumn{3}{|c|}{ Group I (propofol) } & \multicolumn{3}{|c|}{ Group II (etomidate) } \\
\hline & M0 & M1 & M2 & M0 & M1 & M2 \\
\hline $\operatorname{MBP}(\mathrm{mmHg})$ & $93.4 \pm 15.3$ & $78.4 \pm 12.0$ & $83.2 \pm 17.5$ & $97.4 \pm 12.5$ & $81.8 \pm 12.1$ & $94.6 \pm 16.9$ \\
\hline HR (bpm) & $78.2 \pm 15.0$ & $72.2 \pm 12.1$ & $79.0 \pm 15.3$ & $79.0 \pm 12.5$ & $69.2 \pm 14.4$ & $79.3 \pm 21.3$ \\
\hline
\end{tabular}


nitoring on blockade installation. We decided for ulnar nerve stimulation and adductor pollicis response evaluation due to their unique enervation and the similarity of the ulnar nerve-adductor pollicis muscle array with isolated nerve-muscle preparations often used in experimental studies ${ }^{17}$. However, it is important to highlight that, when evaluating tracheal intubation conditions, adductor pollicis monitoring is questionable since the sensitivity of this muscle to non-depolarizing NMBs is different from that of muscles involved in laryngoscopy and tracheal intubation maneuvers ${ }^{18}$.

Most authors correlate the influence of hypnotic agents on neuromuscular block onset to hemodynamic stability and, indirectly, to the degree of muscle perfusion. The hypnotic used for anesthetic induction influences tracheal intubation conditions in different ways: by decreasing laryngeal and pharyngeal reflexes to tracheal intubation stimulations and also by potentiating the effects of some neuromuscular blockers ${ }^{19-21}$

Propofol is widely used to induce anesthesia for significantly depressing laryngeal and pharyngeal reflexes, sometimes doing without non-depolarizing NMBs for tracheal intubation. This evidences the superiority of propofol as compared to other hypnotic agents and is externalized by vocal cords immobility, even seen in studies where non-depolarizing NMBs were not used ${ }^{9,10,22-25}$.

Sparr et al. ${ }^{26}$ have reported acceptable tracheal intubation conditions in $98 \%$ of patients after propofol and rocuronium, and in $80 \%$ of cases with thiopental. Opposite results were reported by other authors ${ }^{27}$, who have found similar results for both agents in terms of tracheal intubation conditions and rocuronium-induced blockade installation.

In our study, tracheal intubation conditions were satisfactory for all propofol group patients and were achieved in a mean time of 48.20 seconds, similar to other studies ${ }^{28}$.

Studies about the influence of hypnotics in neuromuscular blockers onset performed with vecuronium have shown a faster onset with etomidate as compared to thiopental and propofol, which could be attributed to the better hemodynamic profile of etomidate ${ }^{6}$. As to rocuronium, similar results were observed with etomidate and thiopental; however, there has been less diaphragmatic reaction in the etomidate group ${ }^{20}$. In our study, cough and diaphragmatic movement after tracheal intubation were observed with both drugs. This could be explained by the slower onset of rocuronium in the diaphragm as compared to peripheral muscles ${ }^{29}$. All patients were intubated in less than 60 seconds ( 48.20 vs. 51.20 seconds), moment in which neuromuscular block was equal to or higher than $75 \%$ at the adductor pollicis muscle, and previously determined as onset time. The lower incidence of cough in the propofol group (2 patients) as compared to the etomidate group, where 4 patients had mild cough and 5 had moderate cough, may be due to a higher propofol depressing action on laryngeal and pharyngeal reflexes ${ }^{30}$.

As to hemodynamic parameters, despite of the significant MBP decrease after hypnotic administration, it is worth stressing that this had no repercussions on rocuronium onset and neuromuscular block degree, since times for adequate bloc- kade for tracheal intubation and for complete neuromuscular block were equivalent with both hypnotics.

The conclusion is that propofol and etomidate had the same behavior in terms of rocuronium-induced neuromuscular block installation and tracheal intubation conditions.

\section{REFERÊNCIAS - REFERENCES}

01. Mirakhur RK - Dose-response and time-course of action of rocuronium bromide. Eur J Anaesthesiol, 1995;12:(Suppl11): 23-25.

02. Theroux MC, Zgnoev M, Brandom BW et al - Comparison of rapid endotracheal intubations using succinylcholine, mivacurium and vecuronium to combinations of mivacurium and vecuronium. Anesth Analg, 1994;78:S436.

03. Glass PSA, Wlilson W, Mace JA et al - Is the priming principle effective and safe? Anesth Analg, 1989;68:127-134.

04. Braga AFA, Potério GMB - Emprego do pancurônio e alcurônio em doses fracionadas na obtenção de relaxamento muscular para intubação traqueal. Rev Bras Anestesiol, 1993;43: (Supl17):CBA76.

05. Muir AW, Anderson KA, Pow E - Interaction between rocuronium bromide and some drugs, used during anaesthesia. Eur $\mathrm{J}$ Anaesthesiol, 1994;11:(Suppl 9):93-98.

06. Gill RS, Scott RPF - Etomidate short the onset time of neuromuscular block. Br J Anaesth, 1992;69:444-446.

07. Dilger JP, Liu Y, Vidal AM - Interactions of general anaesthetics with single acetylcholine receptor channels. Eur J Anaesthesiol, 1995;12:31-39.

08. Abdel-Zaher AO, Askar FG - The myoneural effects of propofol emulsion (Diprivan) on the nerve-muscle preparations of rats. Pharmacol Res, 1997;36:323-332.

09. Braga AFA, Potério GMB, Braga FSS et al - Intubação traqueal sem relaxantes musculares, utilizando propofol como agente de indução. Rev Bras Anestesiol, 1991;41:(Supl13):CBA130.

10. Braga AFA, Braga FSB, Potério GMB et al - The effect of different doses of propofol on tracheal intubating conditions without muscle relaxant in children. Eur J Anaesthesiol, 2001;18: 384-388.

11. Mallampati SR, Gatt SP, Gugino LD et al - A clinical sign to predict difficult tracheal intubation: a prospective study. Can J Anaesth, 1985;32:429-434.

12. Helbo-Hansen S, Ravlo O, Trap-Andersen S - The influence of alfentanil on the intubating conditions after priming with vecuronium. Acta Anaesthesiol Scand, 1988;32:41-44.

13. Curran MJ, Donati F, Bevan DR - Onset and recovery of atracurium and suxamethonium - induced neuromuscular blockade with simultaneous train-of-four and single twitch stimulation. Br J Anaesth, 1987;59:989-994.

14. Girling KJ, Mahajan RP - The effect of stabilization on the onset of neuromuscular block when assessed using accelerometry. Anesth Analg, 1996;82:1257-1260.

15. McCoy EP, Mirakhur RK, Maddineni VR et al - Pharmacokinetics of rocuronium after bolus and continuous infusion during halothane anaesthesia. Br J Anaesth, 1995;76:29-33.

16. Saxena PR, Dhasmana KM, Prakash O - A comparison of systemic and regional haemodynamic effects of d-tubocurarine, pancuronium and vecuronium. Anesthesiology, 1983;59: 102-108.

17. Ali HH, Savarese JJ - Monitoring of neuromuscular function. Anesthesiology, 1976;45:216-249. 
18. De Mey JC, De Baerdemaeker L, De Laat M et al - The onset of neuromuscular block at the masseter muscle as a predictor of optimal intubating conditions with rocuronium. Eur J Anaesthesiol, 1999;16:387-389.

19. Bali IM, Dundeee JW - Effect of I.V. induction regimens on endotracheal intubation with alcuronium and atracurium. $\mathrm{Br}$ Anaesth, 1985;57:830-831.

20. Fuchs-Buder T, Sparr HJ, Ziegenfuss T - Thiopental or etomidate for rapid sequence induction with rocuronium. $\mathrm{Br} \mathrm{J}$ Anaesth 1998;80:504-506.

21. Skinner HJ, Biswas A, Mahajan RP - Evaluation of intubating conditions with rocuronium and either propofol or etomidate for rapid sequence induction. Anaesthesia, 1998;53:702-710.

22. Keaveny JP, Knell PJ - Intubation under induction doses of propofol. Anaesthesia, 1988;43:S80-81.

23. McKeating K, Bali IM, Dundee JW - The effects of thiopentone and propofol on upper airway integrity. Anaesthesia, 1988;43: 638-640.

24. Scheller MS, Zornow MH, Saidman LJ - Tracheal intubation without the use of muscle relaxants: a technique using propofol and varying doses of alfentanil. Anesth Analg, 1992;75:788-793.

25. Dobson AP, McCluskey A, Meakin G et al - Effective time to satisfactory intubation conditions after administration of rocuronium in adults. Comparison of propofol and thiopentone for rapid sequence induction of anaesthesia. Anaesthesia, 1999;54: 172-197.

26. Sparr HJ, Luger TJ, Heidegger T et al - Comparison of intubating conditions after rocuronium and suxamethonium following "rapid-sequence induction" with thiopentone in elective cases. Acta Anaesthesiol Scand, 1996;40:425-430.

27. Acalovschi I, Bodolea C, Cristea T - Induction with propofol does not improve the intubating conditions of rocuronium. Eur $\mathrm{J}$ Anaesthesiol, 1997;14:(Supp16):13.

28. Crul JF, Vanbelleghem V, Buyse $L$ et al - Rocuronium with alfentanil and propofol allows intubation with 45 seconds. Eur J Anaesthesiol, 1995;12:(Supp11):111-112.

29. Cantineau JP, Porte F, d'Honneur G et al - Neuromuscular effect of rocuronium on the diaphragm and adductor pollicis muscles in anesthetized patients. Anesthesiology, 1994;81:585-590.

30. Guidon-Attali C, Morillac F, Quilichini D et al - Propofol as the main anaesthetic agent in dental surgery. Acta Anaesthesiol Scand, 1990;34:397-399.

\section{RESUMEN}

Munhoz DC, Braga AFA, Potério GMB - Influencia del Propofol y del Etomidato en el Bloqueo Neuromuscular Producido por el Rocuronio. Evaluación por la Aceleromiografia

Justificativa y Objetivos - Algunos hipnóticos pueden interactuar con los bloqueadores neuromusculares (BNM) potencializando sus efectos. El objetivo de este estudio fue evaluar la influencia del propofol y del etomidato sobre el bloqueo neuromuscular producido por el rocuronio.

Método - Fueron incluidos en el estudio 60 pacientes, estado físico ASA I y II, sometidos a cirugías electivas bajo anestesia general, distribuidos aleatoriamente en dos grupos de acuerdo con el hipnótico empleado: Grupo I (propofol) y Grupo II (etomidato). Todos los pacientes recibieron midazolam $(0,1$ $\mathrm{mg} \cdot \mathrm{kg}^{-1}$ ) por vía muscular como medicación pré-anestésica, 30 minutos antes de la cirugía. La inducción anestésica fue obtenida con propofol $\left(2,5 \mathrm{mg} \cdot \mathrm{kg}^{-1}\right)$ o etomidato $\left(0,3 \mathrm{mg} \cdot \mathrm{kg}^{-1}\right)$ precedido de alfentanil $\left(50 \mu \mathrm{g} \cdot \mathrm{kg}^{-1}\right)$ y seguido de rocuronio $(0,6$ $\left.m g . \mathrm{kg}^{-1}\right)$. Los pacientes fueron ventilados bajo máscara con oxígeno a $100 \%$ hasta la obtención de reducción de $75 \%$ o más en la amplitud de la respuesta del músculo aductor del pulgar, cuando fueron realizadas las maniobras de laringoscopia e intubación traqueal. La función neuromuscular fue monitorizada con aceleromiografia. Fueron evaluados: tiempo de inicio de acción del rocuronio $\left(T_{1} \leq 25 \%\right)$; tiempo para instalación del bloqueo neuromuscular total; grado de bloqueo neuromuscular en el momento de la intubación traqueal; condiciones de intubación traqueal y repercusiones hemodinámicas.

Resultados - Los tiempos de inicio de acción e instalación del bloqueo neuromuscular total (segundos) producido por el rocuronio fueron: Grupo I (48,20 $\pm 10,85$ s y $58,87 \pm 10,73 \mathrm{~s}$ ) y Grupo II $(51,20 \pm 13,80$ s y $64,27 \pm 18,55$ s). El grado de bloqueo neuromuscular en el momento de la intubación traqueal fue: Grupo I (77,50\%) y Grupo II (76,96\%). Las condiciones de intubación traqueal fueron satisfactorias en $100 \%$ de los pacientes del Grupo I y en $83,33 \%$ en el Grupo II. En los dos grupos, después de la inyección del hipnótico, se observó disminución significativa de la presión arterial media seguida de elevación.

Conclusiones - El propofol y el etomidato se comportaron de manera semejante en relación a la instalación del bloqueo neuromuscular y a las condiciones de intubación traqueal producidos por el rocuronio. 